\title{
Das globale Dorf - Eine Chimäre
}

\section{Transkulturelle Betrachtungen eines Gastarbeiters im psychiatrisch-psychotherapeutischen Feld}

\author{
Andreas Weichselbraun \\ Psychotherapie-Wissenschaft 8 (2) 31-37 2018 \\ www.psychotherapie-wissenschaft.info \\ CC BY-NC-ND \\ https://doi.org/10.30820/8243.05
}

\begin{abstract}
Zusammenfassung: Der Autor ergründet in diesem Artikel an Hand seiner eigenen Erlebnisse die Herausforderungen des kultur- und sprachübergreifenden Arbeitens im psychiatrisch-psychotherapeutischen Feld in Europa. Dabei stellt er einen Bezug zu den spezifischen Kompetenzen von GestalttherapeutInnen her, wie sie von der European Association for Gestalt Therapy (EAGT) als Modellansatz erarbeitet wurden.
\end{abstract}

Schlüsselwörter: Transkulturelle Psychotherapie, Fremd - sprache, Korzybski, Kernkompetenzen, EAP, Spezifische Kompetenzen, EAGT

\section{Europa im Wandel}

Der öffentliche Diskurs in Europa wurde in den Jahren nach dem Fall des Eisernen Vorhanges vorerst von Öffnung und Integrationswunsch geprägt. Es bestand ein gesellschaftlicher Konsens, die nach dem Zweiten Weltkrieg erfolgte Trennung in Europa überwinden zu wollen und einen Prozess der Zusammenführung einzuleiten. Eine sich daraus allmählich formende Maxime der Europäischen Union, nämlich die der Freiheit der Bewegung, hat einen Migrationsfluss von Ost nach West und von Süd nach Nord, sowohl innerhalb des europäischen Raumes als auch von aussen begünstigt. Dabei hat sich thematisch die Debatte schrittweise von optimistischer Zustimmung in Richtung argwöhnischer Ablehnung des "Anderen» gewandt. Die Offenheit ist dem Misstrauen gegenüber dem Fremden gewichen. Bereits 1989, zur Zeit des Mauerfalls, haben Paul Parin und Goldy ParinMatthey in ihren Sammelband Subjekt im Widerspruch eigene Schriften aus der dem Umbruch vorangegangenen Dekade zusammengefasst, in denen sie unter anderem zu Fragen der Feindbilder des Kalten Krieges und den damit verbundenen inneren und äusseren Grenzziehungen wie auch zum Spannungsfeld zwischen Individuum und Gesellschaft Stellung bezogen haben. Auch gegenüber dem eigenen psychotherapeutischen Berufsstand und dessen Rolle in der Gesellschaft haben sie eine kritische Haltung eingenommen. In dieser Tradition muss es wohl auch jetzt unsere Aufgabe sein, zu hinterfragen, welche Verantwortung wir tragen, wenn es um Fragen von Kultur, eigener und fremder, um Andersartigkeit, um Individualität und Gruppenzugehörigkeit geht. Diese Fragen stellen eine zunehmende Herausforderung an unsere Berufsgruppe dar und im Folgenden versuche ich aus einer persönlichen Perspektive einige dieser Themenkreise anzuschneiden.

Im Jahr 2016 hat das University College London Health Humanities Centre in Zusammenarbeit mit dem
Institute of Advanced Studies eine Kurztagung in London mit dem Titel «Towards Transcultural Histories of Psychotherapies» ${ }^{1}$ durchgeführt, in der einige interessante Aspekte der Wurzeln und der Verbreitung von Psychotherapie diskutiert wurden.

Bei jener erwähnten Tagung ist die These in den Raum gestellt worden, dass die Entwicklung von Psychotherapie ein Ausdruck einer gesellschaftlichen Entwicklung in der «westlichen» Gesellschaft war, die in weiterer Folge in den Rest der Welt exportiert wurde. Bei Sigmund Freud und Fritz und Lore Perls, den Mitbegründern der Gestalttherapie, meiner Modalität, war dies wohl gezwungenermassen der Fall. So betrachtet hat Psychotherapie einen Flucht- und Migrationshintergrund. Gleichermassen wurde auch eindeutig darauf hingewiesen, dass dies kein Einwegprozess war, dass dieser «Export» der Wechselwirkung mit den lokalen Gegebenheiten ausgesetzt war. Letztlich lässt sich dieser Vorgang auch invers betrachten, dass nämlich unser psychiatrischpsychotherapeutisches Handeln sich verändert (und verändern muss), wenn es unterschiedlichen kulturellen Einflüssen, die von aussen an uns herangetragen werden, ausgesetzt wird.

\section{Das Fremde in der Sprache}

Als ich vor nunmehr 15 Jahren den Standort meiner psychiatrisch-psychotherapeutischen Berufsausübung innerhalb Europas von Österreich nach England verlegt habe, war mir zur damaligen Zeit das transkulturelle Arbeiten wohl am ehesten von der Behandlung und Betreuung von Kriegsflüchtlingen aus dem Krieg am Balkan

1 Siehe hierzu: http://www.ucl.ac.uk/institute-of-advanced-studies/ ias-events/towards-transcultural-histories-of-psychotherapies (29.05.2018). 
im stationären Rahmen bekannt. Weder das notwendige Detailwissen um die verworrenen politischen Umstände noch die spezifischen Bedürfnisse der betroffenen Ethnien hat damals im psychotherapeutischen Fachspezifikum oder der Ausbildung zur FachärztIn für Psychiatrie Platz gehabt. Um in der Diktion der Gestalttherapie zu bleiben, war bei mir und meinen KollegInnen eine rasche kreative Anpassung an dramatisch geänderte Feldbedingungen erforderlich. Oftmals geschah dies mit untauglichen Mitteln wie dem Heranziehen von laienhaften ÜbersetzerInnen über die Gräben verfeindeter Ethnien hinweg. Im Versuch ein Verständnis für die innerpsychischen Vorgänge von Kriegstraumatisierten zu erzielen, war dies wohl kein Ruhmesblatt. Der Umstand, dass jemand (annähernd) dieselbe Sprache spricht, wird irrelevant, wenn die DolmetscherIn der verfeindeten Volksgruppe angehört, in ihrem Verhalten nicht ganz unvoreingenommen ist und vermutlich auch gar nicht sein kann. Dennoch haben diese Herausforderungen zumindest eines bewirkt: dass eine gewisse Sensibilisierung im Hinblick auf die kulturspezifischen Unterschiede der PatientInnenschaft stattgefunden hat. Jahre später und zwar im Rahmen der Verlegung meines Arbeitsmittelpunktes nach Grossbritannien sollte ich wieder daran erinnert werden, mit welcher Komplexität wir zunehmend in den seelischen Gesundheitsdiensten konfrontiert werden, wovon die Sprachebarriere nur ein Element ist.

Die Umstellung des psychiatrisch-psychotherapeutischen Arbeitens von der Muttersprache in eine Fremd - sprache ${ }^{2}$ scheint auf den ersten Blick nicht sonderlich kompliziert zu sein, insbesondere wenn es sich um Englisch als Fremd - sprache handelt. Englisch wird nahezu weltumspannend gesprochen und folglich auch gelehrt, oftmals jedoch von Lehrpersonen, deren Muttersprache nicht Englisch ist. So entsteht ein erster Reibungsverlust im Kontakt mit der Sprache, der sich nur sehr schwer aufholen lässt, sowohl im aktiven Sprachgebrauch als auch - was in unserer Berufsgruppe zu den Kernbedingungen gehört - im passiven Sprachgebrauch während des Zuhörens. Darüber hinaus ist Sprache ja nicht nur eine logische Aneinanderreihung von Silben, die entsprechend zusammengesetzt einen beliebig wiederholbaren Sinn ergeben. Sprachlicher Sinn entsteht oft nur kontextuell und ist umgekehrt auch nur im Kontext des Individuums, von dem die Worte, der Satz ausgehen, zu verstehen.

Schon in meinem Vortrag zur D-A-CH-Tagung für Gestalttherapie «Memento mori» in Zürich 2008 habe ich in einem ganz anderen Zusammenhang über das Wort Mutter reflektiert (vgl. Weichselbraun, 2009) und ich möchte dieses Beispiel, weil es besonders eindrücklich ist, wieder heranziehen. Mutter ergibt für jeden einzelnen einen anderen Sinn, nämlich einen individualgeschichtlich verankerten und einzigartigen Sinn. Und dennoch verstehen und verwenden wir das Wort Mutter als einen sehr allgemeinen Begriff. Wenn wir nun versuchen das

2 Bindestrich durch den Verfasser in Anlehnung an Korzybski gesetzt; s. Science and Sanity, 1994, S. ixf.
Wort Mutter mit dem englischen Wort «mother» oder «mum» gleichzusetzen, verschiebt sich diese trügerische Bedeutungsgewissheit grob gesprochen noch um eine weitere Dimension, nämlich die des fremd - sprachlichen Gebrauchs. Wie kann ich meine Mutter als «mother» und «mum» verstehen, wo sie doch nie so eine war? Es war also für mich im Wechsel meines Arbeitsfeldes nicht nur bloss eine Frage, die Aneinanderreihung der englischen Worte - tief eingefärbt vom Yorkshire-Dialekt - zu entziffern und ins Deutsche zu übertragen, ja verstehen zu können, sondern einen darüber hinausgehenden neuen semantischen Sinn abzuleiten, der ein konkretes Arbeiten im psychiatrisch-psychotherapeutischen Sinne erst ermöglicht. $\mathrm{Zu}$ Beginn meiner Tätigkeit schien es mir oft, als hätte ich eine unsichtbare Barriere zu überwinden, als ob mir der Schlüssel zur Erfassung und zum Verständnis der Phänomenologie mit dem unterschiedlichen semantischen Ausdruck im Du des Gegenübers abhandengekommen wäre. Die Worte, das Empfinden in der Begegnung und zuletzt das aufkeimende Verständnis im Kontakt mit dem Du hat einer neuen Stimmigkeit bedurft, für die es noch keinen geeigneten inneren Ausdruck gab. Trotz langjähriger Berufserfahrung kam ich mir manchmal erneut wie ein Berufsanfänger vor. Ich war herausgerissen aus meinem vertrauten Feld, eine Figur ohne sicheren Hintergrund. Andererseits ergab sich dadurch die einmalige Chance, das Gewahrsein neu zu beleben, den neuen Grund zu bereiten, das Verständnis zu erweitern. Im Folgenden möchte ich dies erläutern.

\section{Was Semantik mit Diagnostik in der Psychotherapie zu tun hat}

Fritz Perls hat in seinem ersten, der Gestalttherapie gewidmeten Buch Das Ich, der Hunger und die Aggression (1989 [1942]) sich in seiner Theoriebegründung auf Alfred Korzybski, einen Semantiker, bezogen. In Korzybskis Werk Science and Sanity: An Introduction to Non-Aristotelian Thinking and General Semantics (1994 [1933]) erläutert dieser seine Klassifizierung der Menschen als «time-binding class of life», die vor allem auf der Fähigkeit des menschlichen Nervensystems zur Abstraktion und darüber hinaus zur Symbolisierung der Abstraktion basiert, die zur Entwicklung der Sprache geführt hat. So sehr sich die Sprache als Instrument, «Zeit zu binden» und damit Wissen und Erfahrung weiterzugeben, unerlässlich erwiesen hat, so sehr haben sich die Menschen in der Verwendung der Sprache in kulturspezifischer Weise in ihren Reaktionen «festgefahren». Korzybski bezeichnet das als «semantische Reaktion». In einer Würdigung Korzybskis zitiert Samuel Ichiyié Hayakawa, ein US-amerikanischer Psychologe, zur weiteren Erläuterung den ebenfalls amerikanischen Psychoanalytiker Trigant Burrow:

«Die ursprünglichen Anlagen des menschlichen Organismus werden frühzeitig an das gesellschaftlich bedingte System partieller Reflexe gewöhnt, die wir als den Zeichen-Kodex oder die Sprache bezeichnen; es 
stellt ein System von Reaktionen dar, zu dem der Organismus durch die Sprache erzogen wurde. Daher ist der menschliche Organismus, zusätzlich zu dem ursprünglichen Prozess der Anpassung, von Anfang an weiterer Beeinflussung ausgesetzt [...]. Kurz gesagt wird als Ergebnis seines früheren Trainings das Kind nicht so sehr von einem Feld spontaner, es ganz umfassender und an die Umwelt anpassender Reaktionen eingehüllt, sondern von einem Feld von Ersatzsymbolen, die seine ganze Welt objektiver Wirklichkeit immer mehr ersetzen» (Hayakawa, 1968).

Hineingeworfen in eine fremde Symbolwelt war ich konfrontiert mit einem Gefühl der Verunsicherung, der Sprachlosigkeit. Als ein Klient mir von seiner Verzweiflung berichtete, «that he just lost his pet $\operatorname{dog}$ » («dass gerade sein Hund verstorben war»), so sagte mir das gar nichts. Ich fragte mich sogar, warum er mir das bloss erzähle und was wohl sein wirkliches Problem sei. Der kulturspezifisch erhöhte Stellenwert von Haustieren wirkte be - fremdlich auf mich.

In weiterer Folge waren die Ideen Korzybskis sehr hilfreich für mich, mein Gewahrsein im Einlassen auf die «fremde» Situation erneut zu schulen. Korzybski stellte sich gegen das «Ist der Identität», da es als Feststellung der Identität (absolute Gleichheit in allen Aspekten) den prozessualen Abläufen der Welt nicht entspricht. «Das Wort ist nicht das Ding» (Korzybski, 1994 [1933], S. 60), wie eben Mutter nicht gleich «mom» ist. Um der Komplexität und der Bedeutung der Sprache im menschlichen Er - leben auf den Grund zu gehen, setzte sich Korzybski sehr intensiv mit der Struktur der Wahrnehmung auseinander. Er ging davon aus, dass aufgrund der Struktur und der daraus resultierenden Eigenschaften des menschlichen Nervensystems dieses auf Reize mit einem ihm zugrundeliegenden, system-immanenten Auswahl- bzw. Filterverfahren reagiert. Dieser Selektionsprozess ist vorerst "präattitudinal», das heisst von unseren Vorlieben, Erfahrungen und Stimmungen unabhängig und nur von der individuellen morphologischen Struktur der Wahrnehmenden bestimmt. Im Wahrnehmungsprozess erfolgt ein Umwandlungs- respektive Abstraktionsvorgang, der Aussenreize in Innenreize überführt. Diese Innenreize sind naturgemäss nur ein Abbild der Reizstruktur «da draussen». Die Umwandlung ist von uns ebenfalls nicht beeinflussbar. In einem «vorbewussten»

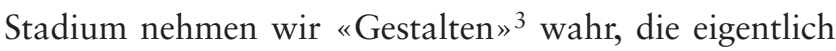
der individuellen Struktur des einzelnen entsprechende Konstrukte sind. Korzybski geht davon aus, dass unsere bewusste Wahrnehmung ein projektiver Vorgang ist, der das Erlebnisereignis nach aussen verlegt, während die Wahrnehmung an sich in Wirklichkeit sich innerlich ereignet. Diese Vorgänge sind jedenfalls nur im Wechselspiel äusserer und innerer Prozesse, einem fortgesetzten

3 «We eventually experience 'gestalts), i. e., central impressions ( feelings, images etc.) constructed multiserially and rapidly from peripheral (environment-organism interface) events» (Pula, 2000, S. 10).
Fluss von Ereignissen in Raum und Zeit, zu verstehen. Wir nehmen darüber hinaus unsere Wahrnehmung, während wir wahrnehmen, in einer Selbstreflexion wahr, was wiederum die gegenwärtigen und - im Sinne einer Erfahrung - auch zukünftige Wahrnehmungsereignisse beeinflusst. Aufgabe sollte es nun sein, sich diesen Vorgang so weit wie möglich bewusst zu machen, sich klar $\mathrm{zu}$ werden, welchen Einflüssen unsere Gestaltbildung und -erkennung ausgesetzt ist und inwieweit semantische Reaktionen dabei eine Rolle spielen.

\section{Fallstudien}

Vor diesem Hintergrund wurde mir in der täglichen Arbeit mit PatientInnen deutlich, wie unabdingbar andauerndes kulturelles Gewahrsein ist, unter anderem auch das Gewahrsein der Bedingungen des eigenen Heranwachsens in einem spezifischen mitteleuropäischen Kontext. Darüber hinaus war und ist die Kenntnis der (Kolonial) - Geschichte des Vereinigten Königreiches hilfreich, um sich in der multiethnischen Vielfalt der englischen Gesellschaft zurechtzufinden.

Im Folgenden will ich an Hand zweier Beispiele aus der Praxis verdeutlichen, dass ein grundsätzliches Wissen um die kultureller Gepflogenheiten anderer Ethnien und differenziertes Verständnis kulturspezifischer Rahmenbedingungen in psychiatrisch-psychotherapeutischer Praxis unabdingbar geworden ist und dass starre Stereotypien keinen Platz haben, da selbst diese Rahmenbedingungen andauernd einem Veränderungsdruck ausgesetzt sind. Bemerkenswert ist, dass diese Begegnungen sich an ein und demselben Tag zugetragen haben.

An diesem erwähnten Tage bin ich zwei Ehepaaren mit pakistanischem Herkunftshintergrund begegnet, in einem Falle einem Paar, dessen Mann anlässlich einer hochfieberhaften Erkrankung seiner Familie eine Angststörung nach massivem Schlafentzug entwickelt hatte, die von einer bizarren Phänomenologie begleitet war. In der Erkundung der frühen Lebensgeschichte seines Heranwachsens in Pakistan kam zutage, dass er seine Mutter, zu der er als jüngster Sohn ein sehr enges Verhältnis pflegte, früh und plötzlich verloren hatte. Bezeichnenderweise litt er selbst zu diesem Zeitpunkt an einer fieberhaften Erkrankung. Seinen Schilderungen zufolge wurde er im Rahmen der Verwirrnisse um den plötzlichen Tod seiner Mutter in seinen eigenen Bedürfnissen völlig missachtet und er erinnerte sich an ein Gefühl der Einsamkeit in einem finsteren Raum. Seine weitere Lebensgeschichte war davon gekennzeichnet, sich von seiner Familie unabhängig zu machen und einen «modernen» Lebensstil einzuschlagen, der insbesondere auch dadurch zum Ausdruck kam, dass er mit seiner Frau eine Liebeshochzeit eingegangen ist, etwas, das nach wie vor noch ungewöhnlich ist bei MigrantInnen aus pakistanischem Umfeld.

Das zweite Paar kam ins Ambulatorium, da die Frau des Ehepaares anlässlich der Reisevorbereitungen zur einer sogenannten kleinen Pilgerreise ('Umra oder Omrah) nach Mekka ein ungewöhnliches Verhalten gegenüber 
Verwandten in der erweiterten Familie gezeigt hatte. Sie hatte sich zunehmend wütend und ausfällig gegenüber männlichen Familienmitgliedern und auch der Mutter ihres Ehemannes geäussert, wüste Beschimpfungen mit Andeutungen von sexuellem Missbrauch und Gewalt ausgestossen, war extrem unruhig und ebenfalls schlafgestört. Auffallend war, dass dieses zweite Paar sich grundlegend anderes präsentiert hat. Der Ehemann, der sich gegenüber seiner Frau sehr fürsorglich zeigte, erwies sich im Gespräch als tonangebend, wohl auch da seine Frau mit ihren Gedanken und Äusserungen sehr von stark wechselnden inneren Vorgängen bestimmt war, doch nicht ausschliesslich so. Vielmehr schien es, als dass er sich seiner traditionellen Rolle als Ehemann und Familienoberhaupt bewusst und gerecht wurde. Den Unterbrechungen seiner Frau mittels Schimpftiraden versuchte er durch Beschwichtigungen und den Wahrheitsgehalt ihrer Aussagen infrage stellend entgegenzukommen. Zweifellos handelte es sich hier um eine eheliche Beziehung, die traditionellen Mustern folgt, bis hin zur Tatsache, dass diese beiden Ehepartner Cousin und Cousine ersten Grades sind und die Heirat arrangiert war. In weiterer Folge wurde der Mann in Haft genommen, da auch gegen ihn der Verdacht des sexuellen Missbrauchs von einem anderen weiblichen Mitglied der Familie erhoben wurde.

Der Kontrast zwischen den beiden Ehepaaren hätte nicht gegensätzlicher sein können: wertkonservativ der Hintergrund in der einen Familie, die Lebensausrichtung auf Individualität basierend in der anderen. Nichts wäre leichter gewesen, bei beiden aufgrund ihrer ungefähren ethnischen Herkunft verallgemeinernd zu ähnlichen Grundannahmen hinsichtlich ihres Verhaltens und ihrer Problemstellung zu gelangen. Wir sind in der alltäglichen Praxis - trotz unseres psychotherapeutischen Bemühens ein Verständnis für die individuelle Situation der KlientIn zur Basis der gemeinsamen Arbeit zu machen - immer wieder dazu geneigt, gewisse ähnliche Abläufe in Gruppen zusammenzufassen, Gemeinsamkeiten zu identifizieren, um eine Erleichterung des Verständnisses und des Arbeitsflusses zu erzielen. Wie die oben angeführten Beispiele verdeutlichen, ist es jedoch unabdingbar, jederzeit dessen gewahr zu sein, dass kulturspezifische Entwicklungsvarianten in Betracht zu ziehen sind und einen deutlichen Einfluss auf die Situation haben können, eben welchen Stellenwert Familie, Individualität und religiöse Orientierung einnehmen.

Dies macht deutlich, dass das psychiatrisch-psychotherapeutische Feld vor allem im europäischen Raum, aber vermutlich weltweit, einer massiven Herausforderung gegenübersteht. Der Begriff des globalen Dorfes greift einfach nicht, ja ist vielmehr irreführend, mag er uns doch eine Vertrautheit vortäuschen, die es nicht gibt. Die Gestalt des Dorfes, wie wir sie semantisch verankert haben als etwas, das einen gewissen Wiedererkennungswert hat, den wir uns im Alltag zu Hilfe nehmen, um die Komplexitäten unseres Alltags vereinfachend zu strukturieren, ist brüchig geworden.

\section{Beschreibung transkultureller Kompetenz in der psychotherapeutischen Lehre und Praxis}

Womit ich vom Persönlichen zum Politischen mit dem Hinweis, dass die psychotherapeutischen Berufsvertretungen dieser Herausforderung allmählich Rechnung getragen habe und sich für eine klare Verankerung transkultureller Kompetenz in Ausbildung und Praxis eingesetzt haben, überleiten möchte.

Die EAP (European Association of Psychotherapy) verabschiedete im Juli 2013 ein Dokument, dass die Kernkompetenzen einer (europäischen) PsychotherapeutIn beschreibt. Domäne 10 dieses Dokuments setzt sich mit den Fragen der Diversität und kulturellen Sensibilität auseinander. Die Formulierungen sind, wie laut Definition des Dokuments zu erwarten, allgemein gehalten, jedoch kann nicht oft genug betont werden, dass mit dieser Festschreibung die Berücksichtigung von transkulturellen Aspekten zumindest auf gesamteuropäische Ebene einen neuen Stellenwert in der Psychotherapie gefunden hat. Die EAGT (European Association for Gestalt Therapy) war noch bis vor Kurzem die einzige psychotherapeutische Modalität, die all ihre Energie darangesetzt hat, diese Kernkompetenzen weiterzuentwickeln, indem sie die speziellen Kompetenzen einer GestalttherapeutIn in einem eigenen umfassenden Dokument darlegte (EAGT, o. J.).

Im Folgenden zitiere ich auszugsweise die Domäne 10 dieses Dokuments, da ich es für einen wichtigen Beitrag in der Debatte zur Professionalisierung der Psychotherapie und damit auch indirekt zur Einbeziehung von kulturellen Aspekten in Ausbildung und Berufsausübung halte. Das Dokument kann in seiner vollen Länge auf der zitierten Webseite eingesehen werden.

\section{«Bereich 10}

Ethische und Kulturelle Sensibilität

Einleitung

Da wir eine zunehmend multikulturelle Gesellschaft werden, werden Unterschiede bezüglich Ethnie und Kultur zwischen Berater und Klient immer wahrscheinlicher (Joyce \& Sills, 2010). Wir müssen uns der kulturellen Vielfalt und anderer kultureller Normen und Werte bewusst sein. In letzter Zeit haben wir uns daran gewöhnt, auf Unterschiede bei Geschlechterrollen in therapeutischen Beziehungen zu achten. Es ist von großer Wichtigkeit, sich des eigenen Geschlechts, der Geschlechtskonstellation in der therapeutischen Beziehung und deren Entwicklung im Verlauf des therapeutischen Prozesses bewusst zu sein. Innerhalb eines europäischen und globalisierten Feldes zu arbeiten, erweitert die Aspekte, die berücksichtigt werden müssen. Es ist sehr wahrscheinlich, dass ein Therapeut KlientInnen annimmt, die von einem anderen kulturellen Hintergrund als er stammen. Dies im Hinterkopf behaltend, müssen wir uns dessen bewusst sein, was Pack-Brown, Thomas und Seymour (2008) über ethische Verantwortung von BeraterInnen betonen, nämlich dass diese professionelle Arbeit leisten müssen, die Respekt vor den kulturellen Ansichten, Werten und Traditionen von 
KlientInnen mit unterschiedlichem kulturellen Hintergrund zeigt. Außerdem stellen Duran, Firehammer und Gonzalez (2008) fest, dass die Kultur ein Teil der Seele ist. Es scheint also, dass die kulturelle Selbstbewusstheit eines Therapeuten sehr wichtig für die therapeutische Reise des Klienten ist (Roysircar et al., 2003; BarYoseph Levine, 2005). Als GestalttherapeutInnen sind wir herausgefordert, uns mit diesen Themen in sich ändernden Bedingungen und Situationen auseinanderzusetzen, da alle Werte grundlegend relativ sind und sich mit Raum und Zeit verändern> (Wertheimer in Lee, 2007). Ein beziehungsorientierter Ansatz zur Ethik betrachtet eine ethische Reaktion auf eine herausfordernde Situation als feldabhängig und muss eine Lösung finden, die <die Entwicklung des Individuums und der Umwelt fördert) (Lee, 2007, in Joyce \& Sills, 2010). Für ethische Dilemmata, Konflikte zwischen zwei oder mehr Werten, die auch als Polaritäten betrachtet werden können, müssen kreative Lösungen gefunden werden, die eine Entwicklung der Beziehung zwischen Individuum und Umwelt ermöglichen, indem authentisch und mit einer Bewusstheit der Beziehungs- und der Feldperspektive gehandelt wird.

\subsection{Eine Gestalttherapeutin/ein Gestalttherapeut ist} mit Folgendem vertraut:

$>$ dem eigenen Hintergrund der Identität und deren Werte bezüglich Ethnie, Kultur und Geschlecht und ist sich klar darüber, dass diese Aspekte die gemeinsam geschaffene therapeutische Beziehung beeinflussen. Eigene kulturelle Glaubenssätze zu entdecken kann für den therapeutischen Prozess sehr wichtig sein (Roysircar, 2004). Aspekte von Unterschieden bei der sexuellen Orientierung, bei Geschlecht, Alter, religiösem Hintergrund, Behinderungen und Bildung müssen berücksichtigt werden.

- Konzepten verschiedener Arten von Ethik, wie normativen und integralen ethischen Themen. Die normative Ethik stellt einen Pol des Feldes dar, der zu akzeptieren ist. Weiter geht es in Richtung der integralen Ethik, zur Betrachtung jedes Pols, und schließlich gibt es die integrale Ethik, wo sich gut und böse, schön und hässlich etc. vereinen. Integration bedeutet, dass wir eine Einheit aus den Teilen bilden, obne einen dieser Teile zu schmälern und seiner Qualitäten zu berauben. Diese Art von Modell bietet der Therapeutin eine Möglichkeit, in der Arbeit mit KlientInnen das inhärente Ungleichgewicht der Kräfte zwischen Therapeutin und Klientin anzuerkennen und mit dem Prozess zu arbeiten, anstatt mit einer Liste von 〈Dos und Dont's ' (Gremmler-Fuhr, 2001, S. 20).

$>$ Dem Konzept, das ethische Themen in der Gestalttherapie als Teil des größeren Feldes versteht und unmittelbar mit einer radikalen Erweiterung der Feldtheorie verknüpft sind. Wheeler (2005) hebt hervor, dass sich das Individuum seine Kultur bereits mit der Geburt aneignet. Laut Reck (2009) sagt Levinas, dass das Individuum, das immer als in Beziehung zu einem Anderen stehend betrachtet wird, an eine ethische Verantwortung der Anderen gegenüber gebunden ist.
$>$ Dem Konzept der organismischen Selbstregulation: Im jeweiligen Hintergrund von Klientin und Therapeutin steht deren eigene, einzigartige Geschichte von Ethnie, Nationalität und Kultur (Joyce \& Sills, 2010) und die Therapeutin ist sich dessen in ihrer Arbeit mit der Klientin bewusst» (ebd., S. 35-37; Herv.d.A.).

Mir ist bewusst, dass dieses Dokument seine stilistischen Mängel hat. Ich habe es dennoch Wort für Wort von der Webseite zitiert und nicht beschönigt. Dadurch kommt ganz besonders zum Ausdruck, dass es ein «Work-inProgress»-Dokument ist, das der ständigen Überarbeitung bedarf, in Anlehnung an die ständigen Veränderungen des Feldes, das es zu beschreiben versucht.

Um wieder zu den beiden pakistanischen Ehepaaren zurückzukehren ist es mir wichtig darauf hinzuweisen, wie viele Querverbindungen in diesem Dokument zwischen ethnischer und ethischer Kompetenz hergestellt werden. Eine einseitige Betrachtung der individuellen Situation dieser zwei Paare, der eigenen kulturellen normativen Ethik folgend, kann zu einer massiven Verzerrung und Nichtbeachtung der verschiedenen Bedürfnisse führen.

So betrachtet fügt sich in den Fallbeispielen einiges zusammen:

- Was verbinden wir mit Begriffen, die als semantische Reaktion «be - fremden», ein Gefühl der Andersartigkeit in uns bewirken, zu raschen Schlussfolgerungen verleiten?

$>$ Wie stehen wir zu andersartigen Wertbildern, insbesondere konservativer Prägung, die definiert durch religiöse Vorschriften den persönlichen Vorstellungen von Gleichheit der Geschlechter, von Individualität und freier Entscheidungsfindung zu widersprechen scheinen?

$>$ Wie sollen wir mit solchen inneren Konflikten auf individueller klientenzentrierter und gesellschaftspolitischer Ebene umgehen?

Als PsychotherapeutInnen sind wir aufgefordert, uns dieser Phänomene besonders bewusst zu sein und Stereotypisierungen kulturellen Verhaltens und Verstehens zu hinterfragen und zu vermeiden. Es ist wichtig und zielführend, der Phänomenologie, in der eine Situation Gestalt annimmt, detailliertes Gewahrsein zu widmen und in weiterer Folge den Prinzipien der organismischen Selbstregulation zu folgen, die wohl am ehesten individuellen als rein normativen Erfordernissen entspricht. Augenmerk kann darauf gelegt werden, welche kulturspezifischen Rahmenbedingungen Kontaktunterbrechungen begünstigen und der organismischen Selbstregulation einen Riegel vorschieben.

Das Kompetenzendokument der EAGT meint dazu erläuternd:

«10.2. Eine Gestalttherapeutin/ein Gestalttherapeut ist interessiert an den Themen der kulturellen, ethnischen und geschlechterbezogenen Diversität und ist fähig:

$>$ zwischen Tugendethik und Prinzipienethik zu unterscheiden: Die Prinzipienethik fragt Ist diese Situation 
unethisch?;, während die Tugendethik fragt <Tue ich das, was das Beste für meinen Klienten ist??. Auch wenn kein ethisches Dilemma vorliegt, verpflichtet die Tugendethik die Professionistin dazu, sich ethischem Verhaltens bewusst zu sein (Meara, Schmidt \& Day (1996), laut Corey, Corey \& Callanan, 2011, S. 41). Eine Gestalttherapeutin integriert beide Aspekte in ibre therapeutische Arbeit, um bessere ethische Entscheidungen treffen zu können» (ebd., S. 37).

Abschliessend möchte ich in diesem Zusammenhang auf einen publizierten Vortrag Umberto Ecos (2012) hinweisen, der im Deutschen den Titel Die Fabrikation des Feindes trägt, in dem er ähnlich zu Parin von der scheinbaren Unausweichlichkeit einer Dämonisierung des Andersartigen in der conditio humana spricht. Angeregt wurde er zu diesem Vortrag, als er am Weg zu einer Veranstaltung in New York von einem Taxifahrer pakistanischer Herkunft gefragt wurde, woher er, Eco, komme. Als dieser ihm sagte, er sei aus Italien, fragte ihn der Taxifahrer, welche Feinde Italien habe, um so offenbar ein bessere Verständnis seiner Herkunft zu bekommen. Dies sein in Pakistan nicht ungewöhnlich, sagte der Fahrer. Be - fremdet hat dies Eco zu dem genannten Beitrag angeregt, der ein historisch fundiertes, wenngleich sehr bedrückendes Menschenbild entwickelt. In der Zusammenschau mit Korzybski, der sein Konzept als Reaktion auf die demagogische Massenpropaganda des Nationalsozialismus entwickelt hat, können die Entwicklungen der letzten Zeit in Europa und wie auch weltweit daher nur sehr bedenklich stimmen. Umso mehr kommt den PsychotherapeutInnen eine Rolle zu, zum gesellschaftlichen Niveau eines differenzierteren Gewahrseins beizutragen. Und das Dokument der EAGT ist dabei eine hilfreiche Erinnerung, sich dieser Herausforderung im Sinne einer berufs- und sozialkritischen Weiterentwicklung zu stellen.

\section{Literatur}

\section{Für den Rahmenartikel}

Eco, U. (2012). Inventing the Enemy. In ders., Inventing the Enemy and Other Occasional Writings (S. 1-21). London: Harvill Secker. European Association for Gestalt Therapy (EAGT) (o. J.). Professionelle Kompetenzen und Qualitative Standards. Spezifische Kompetenzen von GestalttherapeutInnen. http://www.eagt.org/joomla/ images/PDF/Competencies/Comptetences_EAGT_German.pdf (29.05.2018).

European Association of Psychotherapy (EAP) (2013). The Core Competencies of A European Psychotherapist. http://www.euro psyche.org/download/cms/100510/Final-Core-Competencies-v-3-3_July2013.pdf (29.05.2018).

Hayakawa, S.I. (1968). Semantik und verwandte Disziplinen in "Wort und Wirklichkeit». Beiträge zur Allgemeinen Semantik. Darmstadt: Verlag Darmstädter Blätter. http://www.gleichsatz. de/b-u-t/gene/haya2.html\#5 (24.07.2018).

Korzybski, A. (1951). The Role of Language in the Perceptual Process. In R. R. Blake \& G. V. Ramsey (Hrsg.), Perception: An Approach to Personality. New York: The Ronald Press Company. http://com munication.ucsd.edu/_files/Korzybski_Role-of-Language-in-thePerceptual-Process.pdf (03.06.2018).
Korzybski, A. (1994 [1933]). Science and Sanity (5 $5^{\text {th }}$ edition). Englewood/NJ: The International Non-Aristotelian Library Publ. Comp.

Parin, P. \& Parin-Matthey, G. (1989). Subjekt im Widerspruch. Bodenheim: Athenaeum.

Perls, Frederick S. (1989 [1942]) Das Ich, der Hunger und die Aggression. München: dtv, Klett-Cotta.

Pula, R. (2000). A General-Semantic Glossary: Pula's Guide for the Perplexed. Concord/CA: International Society for General Semantics.

Weichselbraun, A. (1999). «... ich verstehe kein Wort». Die Sprache als Phänomen und die Bedeutung von Korzybskis science and Sanity> für die Gestalttherapie. Unpublizierte Abschlussarbeit zur Graduierung in Integrativer Gestalttherapie. Wien.

Weichselbraun, A. (2009). Demenz - ein (un)zeitiger Abschied, eine offene Gestalt? Betrachtungen zu Feldbedingungen und zur Phänomenologie von Demenz an Hand eines Familienschicksals. Gestalttherapie, 23(1), 15-28.

\section{Für den Auszug aus den Spezifischen Kompetenzen von GestalttherapeutInnen (Kapitel 10)}

Amendt-Lyon, N. (2008a). Gender Differences in Gestalt Therapy. Gestalt Review, 12(2), 106-121.

Amendt-Lyon, N. (2008b). Reply to Commentaries. Gestalt Review, 12(2), 140-143.

Amendt-Lyon, N. (2013). Relational Sexual Issues. Love and Lust in context. In G. Francesetti, M. Gecele \& J. Roubal (Hrsg.), Gestalt Therapy in Clinical Practice. From Psychopathology to the Aesthetics of Contact. Mailand: Franco Angeli.

Bar-Yoseph Levine, T. (Hrsg.). (2005). Dialogue Across Cultures. New Orleans: Gestalt Institute Press.

Meara, N. M., Schmidt, L. D. \& Day, J. D. (1996). Principles and Virtues. A Foundation for Ethical Decisions, Policies, and Character. In G. Corey, M. Schneider Corey \& P. Callanan (2011). Issues and Ethic in the Helping Professions. Belmont/CA: Brooks/Cole, Cengage Learning.

Duran, E., Firehammer, J. \& Gonzalez, J. (2008). Liberation psychology as a path toward healing cultural soul wounds. Journal of Counseling and Development, 86(3), 288-295.

Gremmler-Fuhr, M. (2001). Ethic Dimensions in Gestalt Therapy: From a Normative to an Integral Formation of Value Judgments. Gestalt Review, 5(1), 24-43.

Joyce, P. \& Sills, C. (2010). Skills in Gestalt Counselling and Psychotherapy. London: Sage.

Lee, R. G. (2004). The values of Connection. A relational approach to Ethics. New York: Gestalt Press.

Pack-Brown, S.P., Thomas, T.L. \& Seymour, J.M. (2008). Infusing professional ethics into counselor education programs: A multicultural/social justice perspective. Journal of Counseling and Development, 86(3), 296-302.

Reck, M. (2009). The Gestalt of Multiculturalism. An analysis of Gestalt Therapy Theory in Light of Ethnic Diversity with a Focus on Organismic Self-Regulation. Unveröffentlichte Dissertation. School of Professional Psychology. Paper 74. http://commons.pacificu.edu/spp/74.

Roysircar, G., Sandhu, D.S. \& Bibbins, V.E. (2003). Multicultural competencies: A guidebook of practices. Alexandria/VA: American Counseling Association.

Roysircar, G. (2004). Cultural self-awareness assessment. Practice examples from psychology training. Professional Psychology: Research and Practice, 35(6), 658-666.

Ullman, D. \& Wheeler, G. (Hrsg.). (1998). The Gendered Field. Gestalt Perspectives and Readings. Cambridge/MA: GIC Press.

Wheeler G. (2005). Culture, self and field: A Gestalt Guide to the age of complexity. Gestalt Review, 9, 91-128. 
Global Village - A Chimera.

\section{Cross-Cultural observations of a guest worker} in the psychiatric-psychotherapeutic context

The author explores in this article with reference to his own personal experiences the challenges of working across languages and diverse cultural characteristics in the psychosocial field. He relates his findings to the Specific Competencies of Gestalt Therapists as laid down by the European Association for Gestalt Therapy (EAGT) in a model approach.

Key-Words: Cross-Cultural Psychotherapy, Foreign Language, Korzybski, Core Competencies, EAP, Specific Competencies, EAGT

\section{Il villaggio globale - Una chimera.}

Osservazioni transculturali di un lavoratore immigrante nel campo psichiatrico-psicoterapeutico

L'autore in questo articolo insieme alle proprie esperienze espone la sfida del lavoro comprensivo a livello culturale e linguistico in campo psichiatrico-psicoterapeutico. Produce quindi un riferimento alle competenze del terapeuta della Gestalt elaborate dall'European Association for Gestalt Therapy (EAGT) come approccio modellistico.

Parole chiave: psicoterapia transculturale, lingua straniera, Korzybski, competenze di base, EAP, competenze specifiche, EAGT

\section{Der Autor}

Andreas Weichselbraun, Dr. med. univ., MRCPsych, wurde in Villach, Österreich, geboren. Nach Abschluss seines Medizinstudiums an der Medizinischen Fakultät der Universität Wien ist er neben einer Weiterbildung zum Facharzt für Psychiatrie und Neurologie in Gestalttherapie ausgebildet worden. Über die Jahre hat er sowohl in privater Praxis als auch im öffentlichen Gesundheitswesen umfangreiche Erfahrung als Psychiater und Psychotherapeut gewonnen. Seit 15 Jahren arbeitet er im National Health Service in Grossbritannien und ist als Lektor für die Universität Sheffield tätig. Schon während seiner Ausbildungsjahre in Gestalttherapie hat er ein Interesse am Gebrauch und der Bedeutung von Sprache in Psychotherapie wie auch der Diagnostik in der Gestalttherapie entwickelt. Er verfasste mehrere Artikel über medizinische, ausbildungsbezogene und psychotherapeutische Themen. Zwischen 2010 und 2013 war er Redaktionsmitglied der Zeitschrift Gestalttherapie. Er ist Mitglied der European Association for Gestalt Therapy (EAGT).

\section{Kontakt}

Sheffield Health and Social Care NHS Foundation Trust Consultant Psychiatrist-Home Treatment

Northlands Community Health Centre

Southey Hill

Sheffield S5 8BE

a.weichselbraun@outlook.com 